\title{
Médiévales
}

Langues, Textes, Histoire

81 | automne 2021

Voix laïques (XIe-XIIIe siècle)

\section{Leonardo Bigolli Pisani vulgo Fibonacci, Liber Abbaci}

éd. Enrico Giusti, Paolo d'Alessandro, Florence, Olschki (Biblioteca di «nuncius », 79), 2020, cxviii-824 p., $22 \mathrm{pl}$.

\section{Marc Moyon}

\section{CpenEdition}

\section{Journals}

Édition électronique

URL : https://journals.openedition.org/medievales/11965

DOI : 10.4000/medievales. 11965

ISSN : 1777-5892

Éditeur

Presses universitaires de Vincennes

\section{Édition imprimée}

Date de publication : 12 janvier 2022

Pagination : 202-205

ISBN : 978-2-37924-202-1

ISSN : 0751-2708

Référence électronique

Marc Moyon, «Leonardo Bigolli Pisani vulgo Fibonacci, Liber Abbaci », Médiévales [En ligne], 81 | automne 2021, mis en ligne le 12 janvier 2022, consulté le 22 avril 2022. URL : http://

journals.openedition.org/medievales/11965; DOI : https://doi.org/10.4000/medievales.11965

Ce document a été généré automatiquement le 22 avril 2022

Tous droits réservés 


\title{
Leonardo Bigolli Pisani vulgo Fibonacci, Liber Abbaci
}

\author{
éd. Enrico Giusti, Paolo d'Alessandro, Florence, Olschki (Biblioteca di \\ «nuncius », 79), 2020, cxviii-824 p., $22 \mathrm{pl}$.
}

\section{Marc Moyon}

\section{RÉFÉRENCE}

Leonardo Bigolli Pisani vulgo Fibonacci, Liber Abbaci, éd. Enrico Giusti, Paolo d'Alessandro, Florence, Olschki (Biblioteca di «nuncius », 79), 2020, cxviii-824 p., $22 \mathrm{pl}$.

1 Leonardo Pisano (mieux connu sous le nom de Fibonacci) est un mathématicien médiéval qui jouit d'une grande réputation aussi bien chez les historiens que chez les mathématiciens. Paradoxalement, peu d'éléments biographiques peuvent être présentés avec assurance. Le médiéviste regrette naturellement ici le manque de sources le concernant, hormis les informations que le Pisan donne lui-même dans ses travaux, inévitablement sujettes aussi à discussion. Principalement actif dans la première moitié du XIII ${ }^{\mathrm{e}}$ siècle, Leonardo rédige plusieurs travaux couvrant un large spectre des mathématiques. Il donne non seulement à voir une grande connaissance des savoirs et pratiques de son temps avec une évidente appropriation des pays d'Islam, mais il fait aussi preuve d'une maîtrise indéniable des mathématiques aussi bien dans ses aspects définitionnels, ses principes, que dans ses pratiques calculatoires, algorithmiques et hypothético-déductives.

2 Avec l'ouvrage sous recension, Enrico Giusti et Paolo d'Alessandro offrent à la communauté la toute première édition critique du célèbre Liber Abbaci, l'ouvrage le plus connu de Leonardo et le plus important quantitativement. E. Giusti, fin connaisseur du maître pisan, est le principal éditeur de l'ouvrage, P.d'Alessandro ayant rédigé les parties philologiques de l'introduction et révisé l'apparat critique de l'édition. L'ouvrage comble enfin le vide dénoncé par nombre de chercheurs aux demandes récurrentes de disposer d'une édition critique pour ce texte central des mathématiques 
du Moyen Âge. Aussi, l'entreprise éditoriale - techniquement raffinée (livre relié de très grande qualité dans un coffret, avec plusieurs très belles impressions couleur de fac-similé) - doit d'ores et déjà être saluée. Deux parties principales composent l'ouvrage : une courte introduction bilingue (une version italienne précède la version anglaise) reprenant principalement l'historiographie connue (p.xiii-cxvii) et l'édition du texte latin (p.1-690) suivi d'un important et nécessaire appendice critique (p. 691-822).

3 Dans l'introduction, E. Giusti présente d'abord quelques éléments biographiques établis sur Leonardo, tente de replacer l'œuvre du mathématicien dans son temps, notamment au sein de la cour de Frédéric II, et survole le contenu du Liber Abbaci. P. D'Alessandro poursuit avec une partie historiographique revenant sur les anciennes éditions du texte, pour présenter en détail les différents manuscrits connus et utilisés, les relations entre les différents codices, les choix pour établir l'apparat critique, et termine avec deux très courts appendices philologiques (variantes orthographiques et principales lacunes et omissions dans les manuscrits considérés).

4 Rappelant que nous disposons de peu d'informations sur la vie de Leonardo, E. Giusti présente en détail les deux seuls documents contemporains qui sont aujourd'hui connus et déjà étudiés par ailleurs. Le premier est un acte notarié daté du 28 août 1226 dans lequel Leonardo apparaît comme l'acheteur d'un terrain. Le second est un reçu de la ville de Pise pour une pension annuelle versée à Leonardo pour services et travaux rendus à la communauté ; il peut être daté entre 1233 et 1241 et permet d'avancer un terminus post quem pour la mort du Pisan. Complétés d'une étude partielle d'occurrences dans le corpus manuscrit, ces deux documents apportent des informations sur le nom du mathématicien: Leonardo Pisano est complété - de son vivant - par le surnom Bigollo (terme difficile à définir, car tombé en désuétude au plus tard au cours du $\mathrm{XVI}^{\mathrm{e}}$ siècle). Ce n'est qu'au début du XviII ${ }^{e}$ siècle que l'historiographie retiendra le surnom de Fibonacci pour Leonardo Pisano.

5 E. Giusti complète les éléments biographiques par un essai de chronologie de la production scientifique de Leonardo qui prend en compte l'ensemble des textes retrouvés: le Liber Abbaci, la Practica geometriae, le Liber quadratorum, le Flos et l'Epistola ad magistrum Theodorum ${ }^{1}$. Le travail se révèle à nouveau délicat, mais un effort pédagogique est ici réalisé pour faire comprendre les principales preuves et difficultés. Sont présentés des arguments textuels (intercitations, problèmes ou solutions communes) et externes (notamment liés aux relations de Leonardo avec l'empereur Frédéric II qu'il rencontrerait en juillet 1226 et sa cour). Le Liber Abbaci présente deux éditions. La première date de 1202 et semble perdue, à l'exception du chapitre 12 dont l'édition a récemment été publiée par E. Giusti². La date de composition de la seconde édition aujourd'hui admise est 1228, mais, sans nouvel argument, E. Giusti précise explicitement qu'il est nécessaire de considérer avec beaucoup de précautions toute chronologie qui se référait trop précisément à cette année-là. La rédaction de la Practica geometriae remonterait à 1220-1221 sans que cela soit ici remis en cause. Quant aux autres textes, il est difficile de les dater, mais, grosso modo, E. Giusti conclut en considérant la période 1220-1235 comme la période d'activité la plus importante pour Leonardo.

Un résumé laconique du contenu du Liber Abbaci est ensuite proposé (à peine trois pages) renvoyant le lecteur au travail complet de paraphrases en allemand de Heinz Lüneburg ${ }^{3}$. Nous déplorons que les éditeurs n'aient pas fait un autre choix pour que 
l'ouvrage soit réellement autonome. En ce sens, il aurait été intéressant qu'une synthèse récapitulative des différents énoncés soit donnée en annexe pour permettre de mieux saisir le contenu général des quinze chapitres du Liber Abbaci. Ajoutons encore qu'aucune analyse mathématique n'est fournie. Certes, ce travail est colossal étant données la taille et la richesse de l'ouvrage, mais un deuxième volume se concentrant précisément sur le contenu et les mathématiques à l'œuvre dans le Liber Abbaci aurait été apprécié. Les mathématiques de Leonardo peuvent paraître obscures même pour les spécialistes et le seul texte latin ne permet pas de lever toutes les difficultés. Un second volume aurait permis une accessibilité plus importante au texte, pour l'historien (non nécessairement spécialiste des mathématiques), l'historien des mathématiques (non nécessairement latiniste et/ou spécialiste du Moyen Âge), mais aussi pour tout lecteur cultivé et intéressé par l'histoire des mathématiques, par exemple les enseignants. La demande est réelle. Cela aurait encore pu être l'occasion d'indiquer les éventuelles sources de Leonardo, notamment en comparant le contenu du Liber Abbaci avec la tradition textuelle de langues grecque, arabe ou latine (et notamment les nombreuses traductions arabo-latines). Là, les éditeurs renvoient à une bien mince sélection de références bibliographiques à peine signalée.

7 La position de la présente édition critique dans l'historiographie est délicatement amenée en remontant à la première entreprise menée par le jésuite Zaccaria en 1754, avec l'édition de la lettre dédicatoire à Michael Scot et le prologue autobiographique, effectuée à partir d'un seul manuscrit. C'est ensuite au tour de Libri de publier en 1838 une transcription du seul chapitre 15 (principalement dédié à l'algèbre) du même manuscrit dans son Histoire des sciences mathématiques en Italie. Et c'est bien l'édition des écrits de Leonardo réalisée par Boncompagni entre 1854 et 1862 qui fournit les éditions de référence utilisées jusqu'à maintenant ${ }^{4}$. Le travail de l'historien italien est mené de manière scrupuleuse, extrêmement fidèle au seul manuscrit suivi (différent du précédent). Les présents éditeurs précisent que Boncompagni ne semble avoir vérifié aucun calcul pour son édition, regrettant ainsi que le texte soit fautif à de nombreux endroits ${ }^{5}$. Enfin, la toute récente édition du projet de l'Università di Napoli Federico II mené par Giuseppe Germano et Nicoletta Rozza est mentionnée et fortement critiquée pour le manque de compétences mathématiques des membres de l'équipe éditoriale, ce qui les a conduits à plusieurs erreurs de lecture. Ainsi, E. Giusti et P. D’Alessandro promettent une édition fiable aussi bien philologiquement que mathématiquement. Cette promesse semble parfaitement tenue, pour ce que nous avons pu vérifier, même si nous restons convaincu qu'une analyse mathématique des problèmes aurait été profitable.

8 L'introduction s'achève sur une discussion très détaillée de la tradition manuscrite du Liber Abbaci avec la description des vingt manuscrits connus (pour l'édition de 1228, à l'exception d'un témoin, voir supra), pour la plupart déjà connus de Boncompagni. Neuf contiennent tout ou une bonne partie du texte (datés entre le XIII et le xIX ${ }^{e}$ siècle, cinq d'entre eux sont du XIII ${ }^{e}$ ou du XIV ${ }^{e}$ siècle), dix autres contiennent seulement des parties des derniers chapitres (entre le $\mathrm{XIV}^{\mathrm{e}}$ et $\mathrm{XVII}^{\mathrm{e}}$ siècle), un dernier manuscrit appartient à une collection privée, très peu ou pas accessible. La tradition manuscrite montre que le Liber Abbaci aurait pu être originellement conçu en trois volumes : les chapitres 1 à 11, le chapitre 12 puis les chapitres 13 à 15. P. D'Alessandro poursuit en tentant de construire un stemma - avec des arguments principalement philologiques, mais aussi paléographiques extrêmement précis et convaincants -, qui lui permet de justifier 
l'existence d'un archétype et de mettre en évidence deux familles de manuscrits (avec d'éventuelles contaminations) dont une a comme modèle le manuscrit utilisé par Boncompagni.

9 La description des manuscrits et le stemma permettent de justifier les lectures réalisées pour établir l'édition critique, en écartant notamment les manuscrits trop fragmentaires et en privilégiant explicitement six manuscrits pour les chapitres 1 à 11, cinq de ces six pour le chapitre 12, ces cinq mêmes et un septième pour les chapitres 13 à 15. Les choix éditoriaux sont explicitement et clairement présentés : n'est pris en compte que ce qui concerne le texte principal original, les commentaires et les notes marginales ne sont pas traités. Les nombreux diagrammes et autres tables numériques en marge sont fort heureusement conservés et bien référencés dans le texte imprimé. L'édition suit la structure en chapitres de Leonardo. À l'intérieur des chapitres, le texte latin est numéroté par unités textuelles, correspondant aux différents énoncés mathématiques successifs, ce qui en facilite grandement la lecture, le renvoi à l'apparat critique en fin de volume et son utilisation future. On peut néanmoins regretter qu'aucune référence ne soit faite au texte publié par Boncompagni, en rappelant par exemple les pages de l'édition.

En conclusion, Enrico Giusti et Paulo D'Alessandro nous offrent sans aucun doute, avec l'édition critique du Liber Abbaci de Leonardo Pisano, la lecture agréable d'une œuvre médiévale majeure. Tant attendue, cette édition critique, scrupuleuse et techniquement soignée, va très vite devenir l'édition de référence, notamment pour toutes ses qualités philologiques. Cependant, nous disons à nouveau que les éditeurs auraient pu accompagner le texte de différents éléments qui permettraient une meilleure découverte de l'œuvre du mathématicien pisan, comme un travail sur les sources possibles, un index terminologique complet (avec des équivalents grecs et/ou arabes le cas échéant) et bien sûr une analyse mathématique. Fort heureusement, ce travail peut encore être réalisé à partir des matériaux ici rassemblés.

\section{NOTES}

1. E. Giusti revient aussi sur les attributions du Liber minoris guisa - probablement une version courte et remaniée de la première partie du Liber Abbaci - et d'un commentaire au Livre X des Éléments d'Euclide à Leonardo en les considérant comme actuellement perdu.

2. E. Giusti revient aussi sur les attributions du Liber minoris guisa - probablement une version courte et remaniée de la première partie du Liber Abbaci - et d'un commentaire au Livre X des Éléments d'Euclide à Leonardo en les considérant comme actuellement perdu.

3. H. LÜNEBURG, Leonardi Pisani Liber Abbaci oder Lesevergnügen eines Mathematikers, Mannheim, 1993.

4. Scritti di Leonardo Pisano del secolo decimoterzo pubblicati da Baldassarre Boncompagni: Leonardi Pisani Liber Abbaci I. Rome, 1857.

5. C'est sur cette édition que Laurence Sigler s'est appuyé pour établir sa propre traduction anglaise en corrigeant un certain nombre d'erreurs mathématiques, mais en s'éloignant parfois du texte original : L. SIGLER, Fibonacci's Liber Abaci, New York, 2002. Voir, par exemple, la critique 
de M. SPIESSER, «Fibonacci's Liber Abaci. A translation into modern English of Leonardo Pisano's book of calculation », Gazette des mathématiciens, 101 (2004), p. 106-110.

\section{AUTEURS}

MARC MOYON

Université de Limoges 